\section{SERUM ALBUMIN LEVEL PREDICTS INITIAL INTRAVENOUS IMMUNOGLOBULIN TREATMENT FAILURE IN KAWASAKI DISEASE}

H.-C. Kuo ${ }^{1}$, C.-D. Liang ${ }^{2}$, C.-L. Wang ${ }^{3}$, H.-R. Yu', K.D. Yang ${ }^{4}$

${ }^{1}$ Pediatric Allergy and Immunology, ${ }^{2}$ Pediatric Cardiology, Chang Gung Memorial HospitalKaohsiung Medical Center, Chang Gung University College of Medicine, ${ }^{3}$ Pediatric Allergy and Immunology, Po-Jen Hospital, Kaohsiung, ${ }^{4}$ Medical Research and Pediatric Allergy and Immunology, Chang Gung Memorial Hospital-Kaohsiung Medical Center, Chang Gung University College of Medicine, Kaohsiung, Taiwan R.O.C.

Objectives: Kawasaki disease (KD) is a systemic vasculitis primarily affecting children who are less than 5 years old. Intravenous immunoglobulin (IVIG) is the standard therapy for KD. However, many KD patients still show poor response to initial IVIG treatment. This study was conducted to investigate the risk factors for IVIG treatment failure in KD.

Methods: Children who met KD diagnosis criteria and were admitted for IVIG treatment were retrospectively enrolled for analysis. Patients were divided into IVIG-responsive and IVIG-resistant groups. Initial laboratory data before IVIG treatment were collected for analysis.

Results: A total of 131 patients were enrolled during the study period. At $48 \mathrm{~h}$ after completion of initial IVIG treatment, 20 patients (15.3\%) had an elevated body temperature. Univariate analysis showed that patients who had initial findings of high neutrophil count, abnormal liver function, low serum albumin level $(<2.9 \mathrm{~g} / \mathrm{dL})$, and pericardial effusion were at risk for IVIG treatment failure. Multivariate analysis with a logistic regression procedure showed that serum albumin level was considered the independent predicting factor of IVIG resistance in $\mathrm{KD}$ patients $(P=0.006$, OR $=40,95 \% \mathrm{Cl}$ : 52.8 562). There was no significant correlation between age, gender, fever duration before IVIG treatment, hemoglobin level, total leukocyte and platelet counts, C-reactive protein level, or sterile pyuria and initial IVIG treatment failure. The specificity and sensitivity for prediction of IVIG treatment failure in this study were $96 \%$ and $34 \%$, respectively.

Conclusion: Pre-IVIG treatment serum albumin levels are a useful predictor of IVIG resistance in KD patients.

\section{SINCOPES IN CHILDREN AND PARTICIPE CARDIAC DISEASES IN ETIOLOGY}

\author{
Z. Inić, Paediatrics Cardiology \\ Cardiology, Institute of Childs Disease, \\ Podgorica, Montenegro
}

Sincope is a sudenly, transientloss of consciousness, caused by interruption of essential energy substrates to the brain. Some of the reasons are different heart diseases, which cause a sudden fall in cardiac output.There are usually during exertion, and need urgent treament.

Aim was to show participation heart diseases in etiology sincope in pediatric patients reffered to our clinic between 2004-2008.y.

Methods: This study includes 153 children, with one or repetitive sincopes, without patients with clear clinic presentation for neurologic sincope. In all patients we have done physical examination of the cardiovascular system and surface ECG. Some patients have undergone echocardiography, ambulatory 24hour(Holter) ECG and exercise testing.

Results: The most of all examined patients, $106(62,09 \%)$, had neurally mediated reflexive and orthostatic sincope. The most of them are teenagers. Six patients with neurally mediated sincope have mitral valve prolapse, without arrythmias.In $32(20,91 \%)$ we havenct founded etiology, but we excluded heart diseases and $17(11,11 \%)$ of them have neurologic sincope probably.But only 4 patients $(2,61 \%)$ have diagnosed heart disease. One patient have VT exertion related, another patient have cardiac tumor (right ventricle). Third patient have aoric valve stenosis, grade middle to severely.And finally with congenital long QT syndrome.

Conclusions: Sincope is one of the most often reason for referreing patients to cardiologist in our clinic.But small number of them have heart disease, and always seriosly. The reason is probably good organisation pediatric and cardiology services and discovering heart diseases in early stage, with following and treatment in time. 